\title{
AN INVESTIGATION ON SOFT MAGNETIC AND NON- MAGNETIC MATERIALS UNDER LOW FREQUENCY FOR BIOMEDICAL SENSOR APPLICATION
}

\author{
Atika Arshad, Sheroz Khan, AHM Zahirul Alam and Rumana Tasnim \\ ECE Deptartment, Faculty of Engineering, International Islamic University Malaysia (IIUM), \\ Jalan Gombak, 53100 Kuala Lumpur, Malaysia. \\ sheroz@iiu.edu.my
}

\begin{abstract}
In consequence of the recent development of magnetic sensors in biomedical sector, the investigation of magneticmaterials has been a contributing factor in application stage. This paper proposes a novel technique to investigate materials by obtaining unique distinctive impedance peaks with unique impedance values. A magneto-inductive sensoris used to measure the induction of magnetic and non-magnetic impedance peaks related to the change in permeability, thus characterizing the materials under low frequency.
\end{abstract}

KEY WORDS: Inductive transducer, magneto-inductive, inductor, Inductive Element under Test (IEUT), Resistive Material Under Test (MUT).

\section{INTRODUCTION}

We are now passing through a period of time when soft magnetic materials are getting increased attention from researchers in the study of materials. Magnetic materials are of essential importance in many industrial and engineering applications and have been researched for a long time. Over the past years soft magnetic magnets were only being used for industrial purposes, but now soft magnetic magnets have become an active and dynamic expression of research interest for its numerous ranges of applications in biomedical applications. With the help of the knowledge of materials, today's research focuses on new technologies like nanostructured magnetic materials to achieve specific properties desired for its applications. The integration of nano-technology in the medical field continues to revolutionize the instruments used in hospital care.

Formerly surgery used to be carried out on human body to replace the batteries. At present contactless means has been implemented, using an external energy source to charge batteries for electronics in a human body. This system generally includes a wireless power transmitter and receiver coupled together through inductive means (non-contact) means to supply energy to the implanted devices within a human body, thus making it safer for patients from getting infected.

Wireless sensor medical experts are able to intelligently monitor medical condition of patients like detecting and monitoring the respiratory system, the contraction of blood vessels, cardiac pressure disorders etc. A sensor such as the heart rate sensor takes an 
electrocardiogram reading from the heart rate of a patient and the sensor then relays the collected data to a computer, where the patient is monitored concurrently.

In the human body, ions continually move within and outside the cells. The electrical activity inside human body is responsible for producing magnetic fields which is generally measured using sensitive instruments. To be more specific, the technology of magnetic field sensing has been vastly widened as today's biomedical field has adapted a variety of magnetic sensors to detect the presence, strength, or direction of magnetic fields from permanent magnets, magnetized soft magnets, vehicle disturbances, brain wave activity or fields generated from electric currents. Magnetic sensors are capable of measuring these properties by noncontact means. Fig. 1 shows a basic block diagram of a magnetic sensor to detect direction, presence, rotation, angle, and electrical currents in a specific way, theses sensors detects the changes brought about in magnetic field, the output signal obtained will be processed in order to translate the sensor output into the desired parametric value. Hence magnetic sensing is used in numerous applications for its reliable and accurate sensing of parameters of interest.

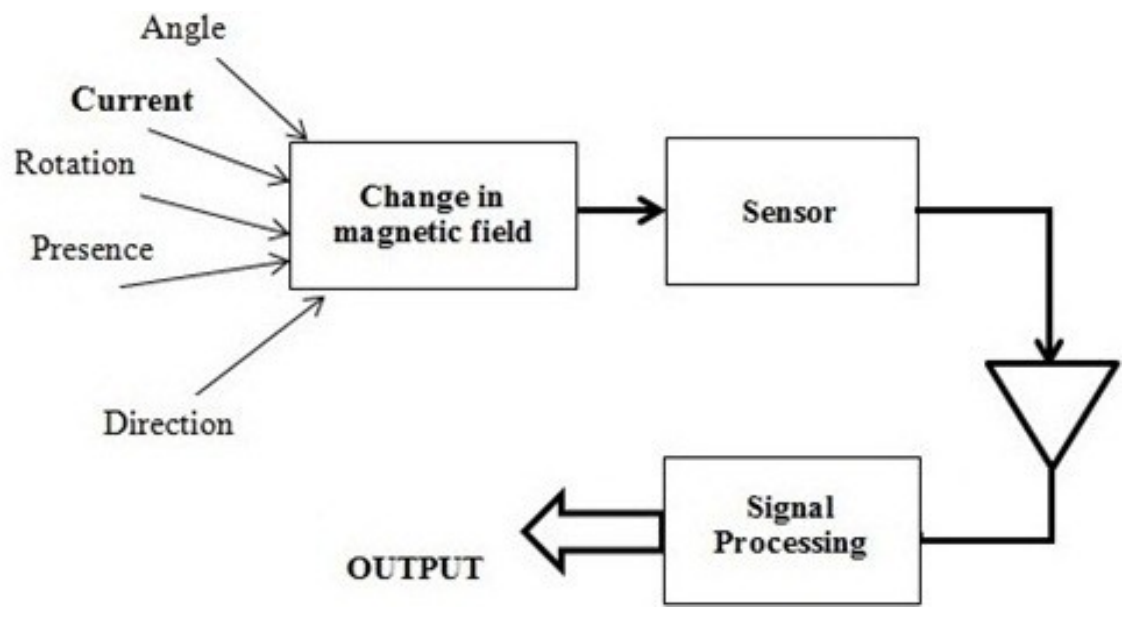

Fig. 1: Block diagram of a typical magnetic sensing system

In these magnetic sensors, magnetic materials generally surround the sensor elements and are used as in the form of coil in transducers and magnetic sensors. These sensing elements come under the category of inductive sensing [1-2]. The ongoing development of different sensors has called for the noteworthy improvement for bio-implantable devices, medical equipments and also for a wide range of applications. Therefore such magnetic properties including magnetic materials should be inspected. A numerous range of new applications are being added and developed due to the availability of electromagnetic material, superconductive magnetic materials and permanent magnetic materials. For understanding the function of different sensors under various conditions, users should have prior knowledge of materials properties. The primary focus of this work is to study soft magnetic materials at low frequency in applications where reliability is the main issue of concern compared to faster response. These responses are triggered as a result of measuring parameters such as resistivity and permeability. In quite a number of applications, the materials triggering responses are to be identified using their specific properties. 
Extensive research[3-6] have gained interests on magnetic induction of magnetic materials for its' wide range of application.Magneto-inductive sensors are used in the applications like electronic stethoscope, ECG machine etc. Magneto-inductive effect observed in soft ferromagnetic materials have been used to design various sensors whereas ferromagnetic materials have been studied since several years which have only found its' applications in recent times. Past research [7] has designed a tongue drive system for enabling a person with severe disabilities. It determines the users' intentions by tracking the movements of a permanent magnetic tracer wirelessly that is secured on their tongue using an array of magnetic sensors. For homogenous magnetic field detection, the GMI magnetometers have been implemented [8] by noise evaluation technique. In the magnetoinductive sensor the alternating current (ac) impedance of a soft ferromagnetic material alters with the longitudinal component of a direct current (dc) magnetic field.

An inventive approach for the modeling of ferromagnetic targets are presented [8] where the magnetic sensors have been used for target detection, localization and tracking witha wireless sensor network (WSN). The authors have used experimentally validated magnetic signal models and evaluated the sensitivity and energy efficiency of proposed technique.

The development of various sensors has opened up several possibilities for significant improvements in the advancement of bio-implantable devices or medical equipments. Hence many problems regarding magnetic properties exist to be examined from the elementary viewpoint. To select the magnetic material and be completely familiar with the characterization of its' magnetic properties proper investigation is required. Aneurysm clip is an implant used for closing blood vessels located in hardly accessible regions of patients' body. It generally undergoes magnetic resonance (MR) procedures in MR systems. The previous research [9] of this particular implant emphasized the significance in evaluating the relative amount of magnetism present in the aneurysm clip. It is found that only the patients or individuals with non-ferromagnetic or weakly ferromagnetic clips are allowed into the MR environment. Hereby the identification of materials appears to guide in using for suitable applications.

Besides, magnetic materials are major components to construct cost effective wireless passive sensors like magneto-elasticsensor and the magneto-harmonic sensor which are widely used to examine chemical concentrationssuch as glucose, ammonia, carbon dioxide, and $\mathrm{pH}$ by applying a mass- and/or elasticity-changing chemicalresponsivelayer [10]. Under an AC magnetic field excitation, these sensors resonate at their resonant frequencies. The relative amount of the magnetic materials and the characteristics of these material properties should be taken into consideration. In this paper, we emphasized on a method to characterize the materials and as a part of experiment we took four materials. This method can be used in case of above mentioned wireless passive sensors as both the sensing and biasing elements of these sensors are magnetic materials.

This work proposes a novel technique for investigation of soft magnetic and nonmagnetic materials under low frequency using analytical approach based on circuit analysis theory. The proposed work aims at identifying proper magnetic material to be used as core of inductive sensory transducers, ultimately identifying them for sensors fabrication, thus differentiating the materials by gaining distinctive impedance peaks with unique impedance values. Design specifications of the proposed model includes the sensing circuit and appropriate simulation results. 


\section{PASSIVE REACTIVE CIRCUIT ELEMENT THEORY}

It is desirable for implantable bio-medical devices to have wireless communication. Therefore, wireless inductive powering is a reliable solution based on inductive coupling between the transmitter coil placed outside the body and the implanted receiver coil inside the body. A Basic inductive concept is explained for the coil design.

A resistive transducer is a device that senses a change in the parameter of interest as a change in the resistance. A common type of resistive transducer is the resistive displacement transducer; it uses a resistive element with a sliding contact linked to the object the position of which is being monitored.

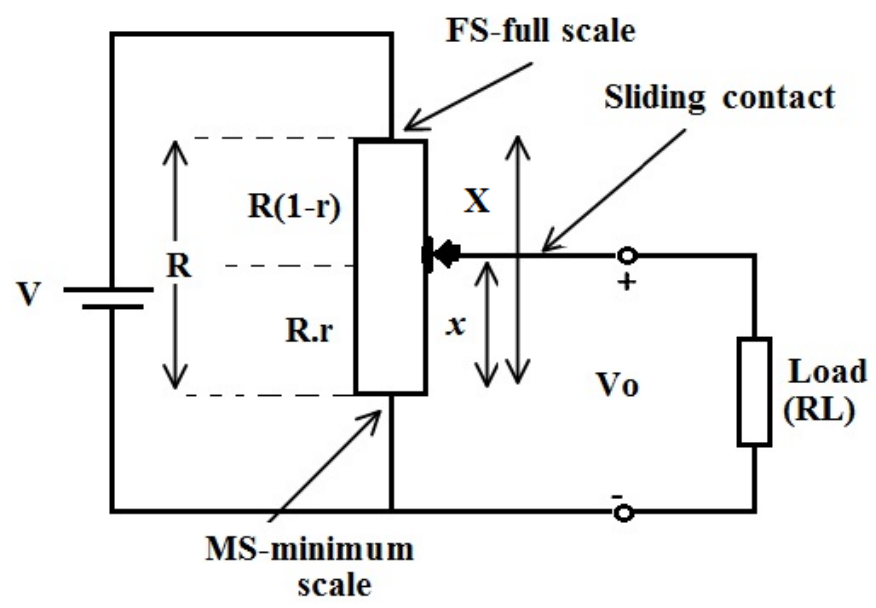

(a)

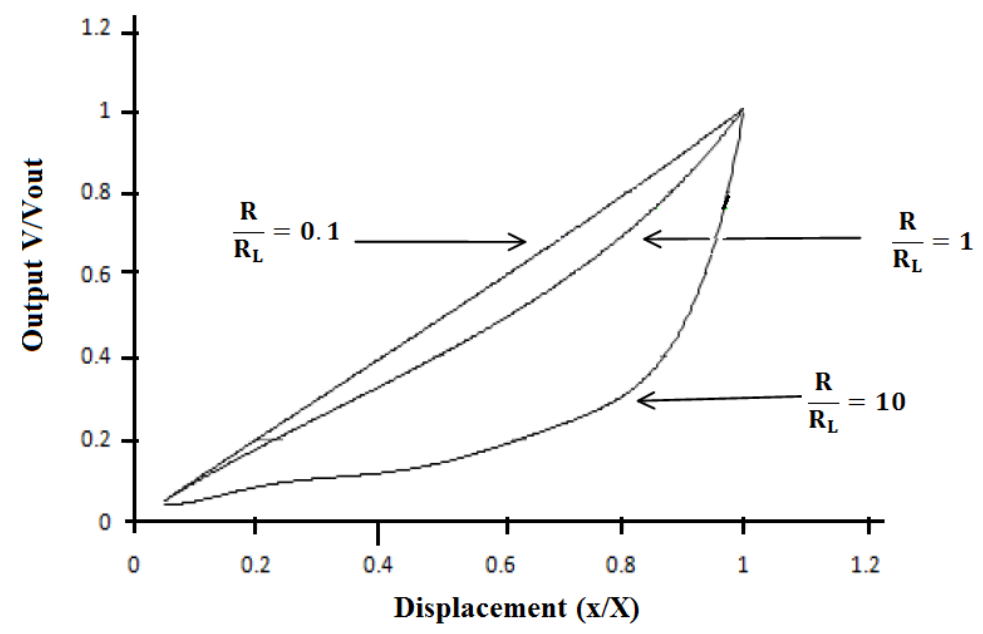

(b)

Fig. 2: (a) Basic resistive transducer, (b) Output of resistive transducer [13] 
The basic working principle of the resistive position transducer is that the measured quantity causes a resistance change in the sensing element. The output voltage (Vout) across the sliding contact exhibits a linear response to changes in displacement under noload $\left(\mathrm{R}_{\mathrm{L}} \rightarrow \infty\right)$ conditions (Eq.1). However, the response does not remain very linear under specific load conditions, as shown in Fig.2, where the sliding contact of variable resistance (Potentiometer) slides on rail of length $(\mathrm{R})$ with the purpose of maximizing or minimizing resistive value (RL). This results in controlling output voltage (Vo) accordingly.

$$
V_{\text {out }}={ }_{K}^{n}=
$$

The linearity of the resistor is affected badly by the ratio of the load resistance, $\mathrm{R}_{\mathrm{L}}$, to potentiometer resistance, $R$.

$$
\frac{V_{\text {out }}}{V}=\frac{\left(\frac{x}{\cdots}\right)\left(\frac{R_{L}}{R}\right)}{\left(\frac{\kappa_{L}}{R}\right)+\frac{-}{x}-x}
$$

where ' $x$ ' represents the sliding contact position and ' $X$ ' is the maximum position of the slider. The change in resistance due to the variation in the net magnetic flux is what's required to be measured. This analysis is further carried out in Chapter 4 to see the effect of resistor on magnetic materials, thus characterizing the materials.

Capacitive transducer sensors works by measuring changes in an electrical propertycalled capacitance.Capacitance is described by measuring the output voltages across a conductor. The amount of current flow is determined by the capacitance, and the capacitance is determined by three parameters: (a) capacitance changes with changes in area; (b) capacitance changes with changes in distance; and (c) capacitance changes with changes in dielectric, where the capacitance is directly proportional to the surface area of the objects and the dielectric constant of the material between them, and inversely proportional to the distance between them.

$$
C(x)={ }_{x}^{-}=
$$

where,

$\mathrm{C}=$ capacitance of the capacitor

$\varepsilon=$ dielectric constant or permeability

$\varepsilon_{r}=$ relative dielectric constant

$\varepsilon_{o}=$ free space dielectric constant

$x=$ distance between the plates [m]

$A=$ area of the plates $\left[\mathrm{m}^{2}\right]$ 
A change in any of the three parameters leads to change in capacitance, which is a measure of the quality to be measured. Hence the capacitive transducer is able detect the presence of metallic element without contact and indicates their presence by variations in its capacitive values.
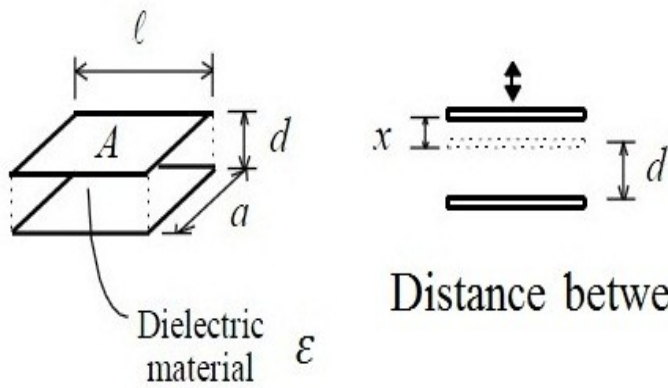

Distance between the plate

Change in area

Fig. 3: Changes which can affect capacitive transducer (Area of the plate, distance between the plate, and type of dielectric)

Inductive transducer represents a hollow solenoid winding with core made of the material under test as illustrated in Fig. 4, the solenoid consisting of primary coil and secondary coil. When a core made of materialswhich are being tested is moved in and out continuously at a given frequency, it leads to producing a change in the inductance and hence a change in the corresponding output. The output from the circuit is calibrated directly against the value of the input such that it becomes a representative of the material the core is made of. The inductance to be measured is dependent on the nature of materials $\left(\mu_{\mathrm{r}}\right)$.

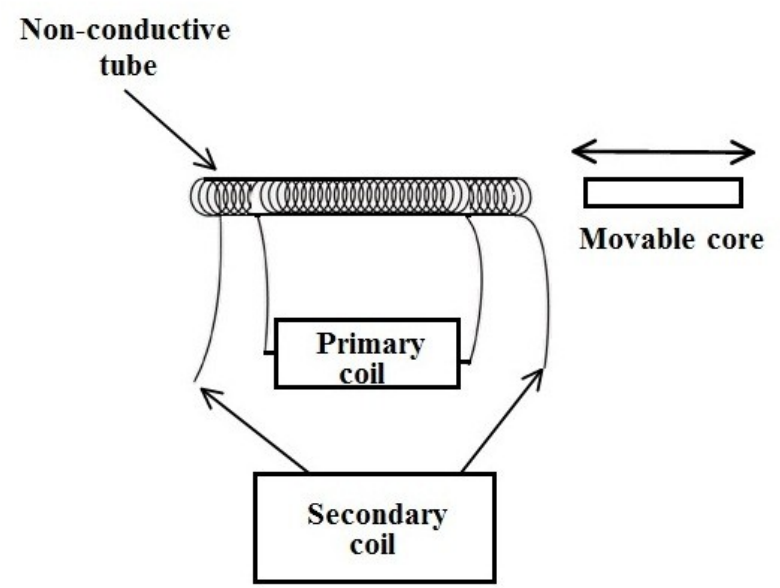

Fig. 4: Material testing coil 


\section{CIRCUIT ANALYSIS}

Fig.5 is used for obtaining the theoretical and experimental results in this work. This model circuit is integrated with three inductive circuits: excitation circuit (marked A), test circuit (marked B), and read-out circuit (marked C). This whole setup makes up a noncontact method of characterizing materials, which is based on inductive coupling for energizing the Inductive Element Under Test/ Resistive Material under Test with cores made up of the materials being characterized. A set of two experiments were carried out (see Fig.6), the first experiment was done with Inductor for two set of coils, and the second experiment was done by replacing the inductor with resistor of two different resistive values. A series of graph was plotted, then analyzed based on their unique peak values.

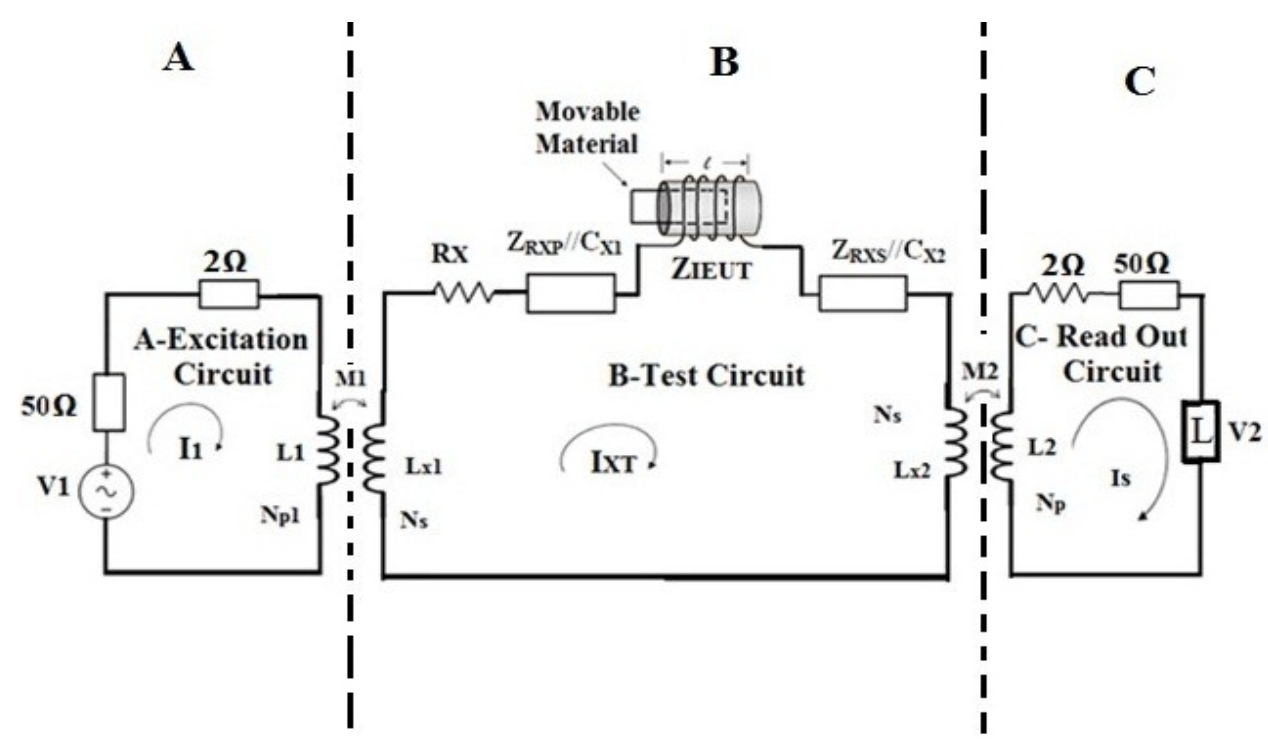

Fig. 5: Schematic model of the circuit diagram

A- Excitation circuit provides an external power supply, which is connecting to the test circuit by non-contact means that is through inductive coupling. There exists a mutual inductance $M_{1}$ between the loops. The signal source circuit has an impedance of $Z_{1}=++$.

B- Test circuit is the transducer processing circuit, connected to both the excitation circuit and the read-out circuit through inductive coupling. To enable the measurement without direct electrical contact, two inductive couplings are used $L, L_{1}$ and $L_{2} . M_{1}$ is the mutual inductance which exists between the excitation circuit and the test circuit, and $M_{2}$ is the mutual inductance existing between the test circuit and the read-out circuit. $Z_{R X P} \| C_{X 1}$ and $Z_{R X S} \| C_{X 2}$ is the parallel impedance of the fixed resistor $\mathrm{R}$ and the bypass capacitor $C_{X 1}$ and $C_{X 2}$ respectively. $C_{X 1}$ and $C_{X 2}$ serves as the low impedance bypass capacitors for the coupled AC signal. The 
transducer processing circuit, has a transducer impedance $Z_{I E U T}$, self-impedance $R_{X}+$, and reflected impedances $Z_{R X P}$ and $Z_{R X S}$.

C- Readout circuit is the signal measuring circuit which measures $V_{2}$, the resultant signal voltage produced when a material is inserted in the IEUT coil.

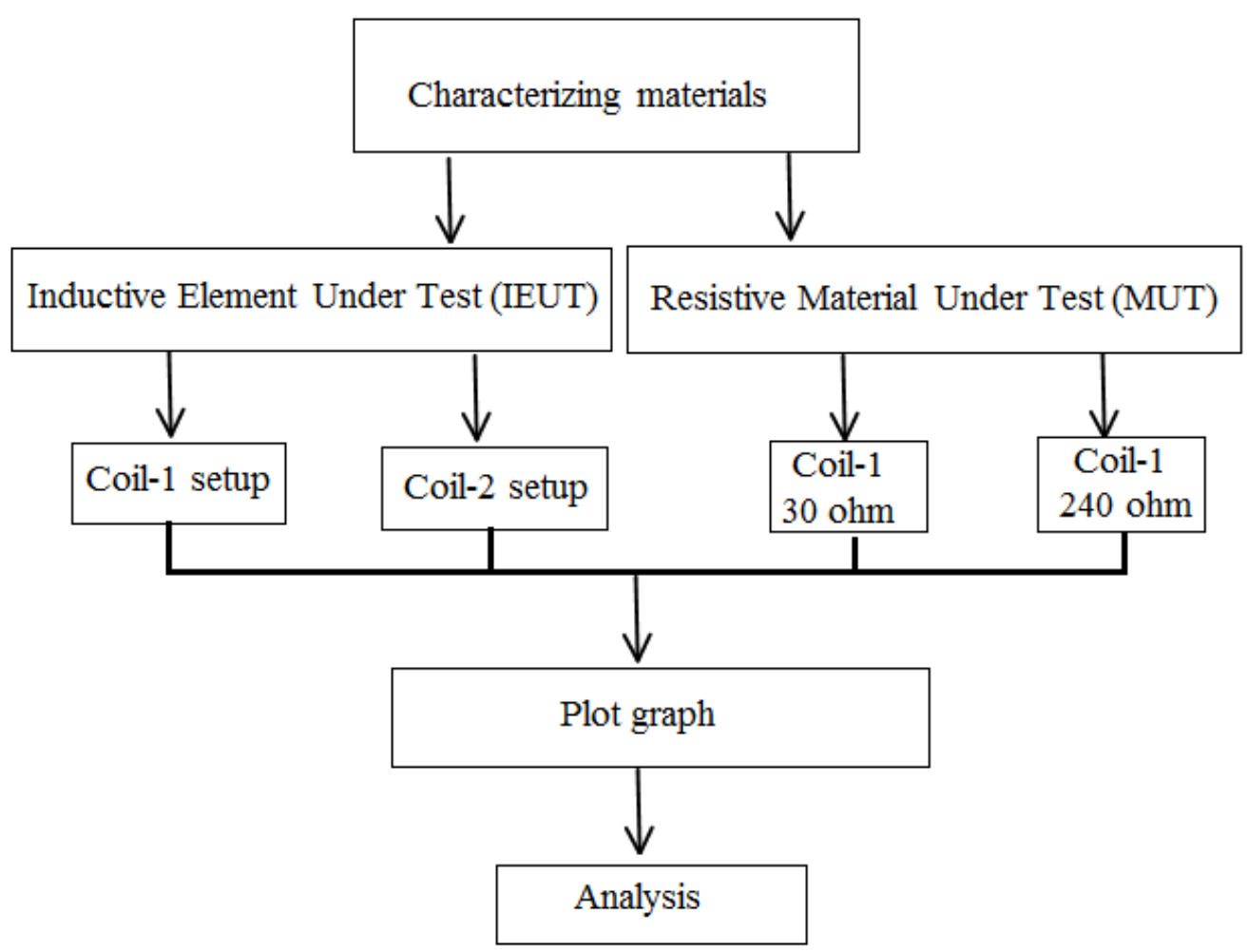

Fig. 6: Flowchart diagram of the experimental methods

Here $Z_{I E U T}$ is a function of change in inductance.

$$
I_{X T}=j \omega+\frac{V_{M X P}}{+\quad++}
$$

Where, $V_{M X P}$ is the voltage inducedto the circuit is;

$$
V_{M X P}=\stackrel{i}{K_{1}+}-
$$

The reflected impedance given by the transmitting signal is;

$$
Z_{R X P}=\kappa_{1}+-i
$$

The reflected impedance received is given by; 


$$
Z_{R X S}=\underset{K_{2}+}{\cdots}-I
$$

The above expressions are used to derive the current $I_{s}$ and hence,

$$
V_{2}=
$$

where,

$$
I_{s}=\frac{\cdots}{K_{2}+}-
$$

An expression for $I_{X T}$ is derived as shown in Eq. (4), using the circuit in Fig. 5 as a function of $Z_{I E U T}, Z_{R X P}$ and $Z_{R X S}$, keeping all other constant. Therefore, the current $I_{X T}$ can be written as:

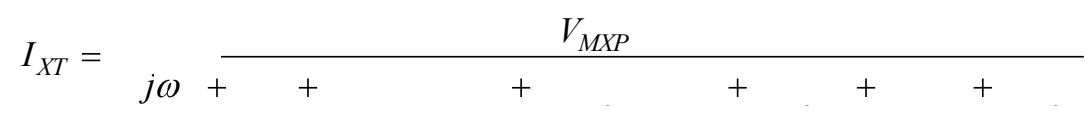

Since the impedance of IEUT ( $\mathrm{Z}_{\text {IEUT }}$ ), is the function which changes with inductance is of interest, the other impedance can be grouped together as Zsetup, where

$$
Z_{\text {setup }}=\quad+\quad+\quad+\quad+\quad+\quad+2
$$

Moreover (5) can be simplified as shown.

$$
I_{X T}={ }_{L_{\text {setup }}+}^{V}
$$

Hence,

$$
Z_{\text {IEUT }}={ }_{1_{X T}}^{V}-\cdots
$$

The induced current $\mathrm{I}_{\mathrm{XT}}$ measured by the secondary side is expressed as;

$$
I_{X T}={ }^{V}
$$

where $Z_{C I}$ is the calibrated impedance response [5], it is defined as the ratio of the voltage measured from secondary circuit and the current flowing through the secondary circuit. Then, E.q. (9) is obtained by substituting $Z_{I E U T}={ }_{I_{X T}}{ }_{I_{X}}-\ldots$ and $V_{M X P}={ }_{K_{1}+}-$, as follows,

$$
\begin{aligned}
& Z_{\text {IEUT }}={ }_{\frac{r_{2}}{Z_{C I}}}^{r}-. \\
& Z_{\text {IEUT }}=\underset{K_{1}+}{i \ldots} \quad{ }^{-} \\
& \text {Let } k=k_{1}+\ldots \text {, and }
\end{aligned}
$$




$$
Z_{\text {IEUT }}={ }_{V_{2}}^{l_{I} T}-\ldots
$$

In order obtain the values of $\mathrm{Z}_{\mathrm{IEUT}} / \mathrm{Z}_{\mathrm{MUT}}$, the two unknown parameters ( $\mathrm{k}$ and $\mathrm{Z}_{\text {setup }}$ ) must be determined first. The value of $K$ and $Z_{\text {setup }}$ is computed by multiplying the frequency used with the value of inductance and solving two simultaneous equations. For instance, the first frequency is taken to be $10 \mathrm{kHz}$, value of inductance used is $4.5 \mathrm{mH}, \mathrm{V}_{1}$ is set to constant voltage of $3 \mathrm{~V}$ and $\mathrm{V}_{2}=0.82 \mathrm{~V}$ is the output voltage values obtained. By using Eq. (9),

The calculation for the data is as follows;

$$
\begin{aligned}
& Z_{\text {IEUT }}= \\
& 10 \mathrm{kHz} X 4.5 \mathrm{mH}=\mathrm{K}(3 / 0.82)-\mathrm{Z}_{\text {setup }} \rightarrow \text { (i) } \\
& 12 \mathrm{kHz} \text { X } 4.5 \mathrm{mH}=\mathrm{K}(3 / 0.86)-\mathrm{Z}_{\text {setup }} \rightarrow \text { (ii) }
\end{aligned}
$$

Solving these two equations simultaneously, the value of $K=-52.63$ and $Z_{\text {setup }}=-238.5$. Thus, the values of $\mathrm{K}$ and $\mathrm{Z}$ are inserted in Eq. (9), to obtain a series of results, which is then plotted for inductance against frequency.

\section{EXPERIMENTAL SETUP}

The technique was implemented based on the experimental setup shown in Fig.7. The equipment used for both the experimental setup included the function generator, oscilloscope, volt-meters and cored coils. The function generator provides the external power needed to excite the primary coil. In the experiment, $V_{1}$ was set with an input of $V_{p p}=\quad$ with frequencyranging from $1 \mathrm{KHz}$ to $45 \mathrm{KHz}$.To obtain experimental results, one of the coils was excited by a signal at a given frequency, which was received and measured through another coil. Measurements were recorded across the resulting coupled signal in the circuit loop from the read-out circuit. The output value $V_{2}$ was recorded for every frequency increment of $1 \mathrm{KHz}$. The inductive transducer coil (IEUT/MUT) is the subject under test. Several different materials were used for the test; steel, plasticand air/paper core (used as a reference). Two sets of experiment were carried out under similar experimental procedures with two different sets of coil. The electrical specification of the coils used in these two experiments is given in Table 1 and Table 2. 


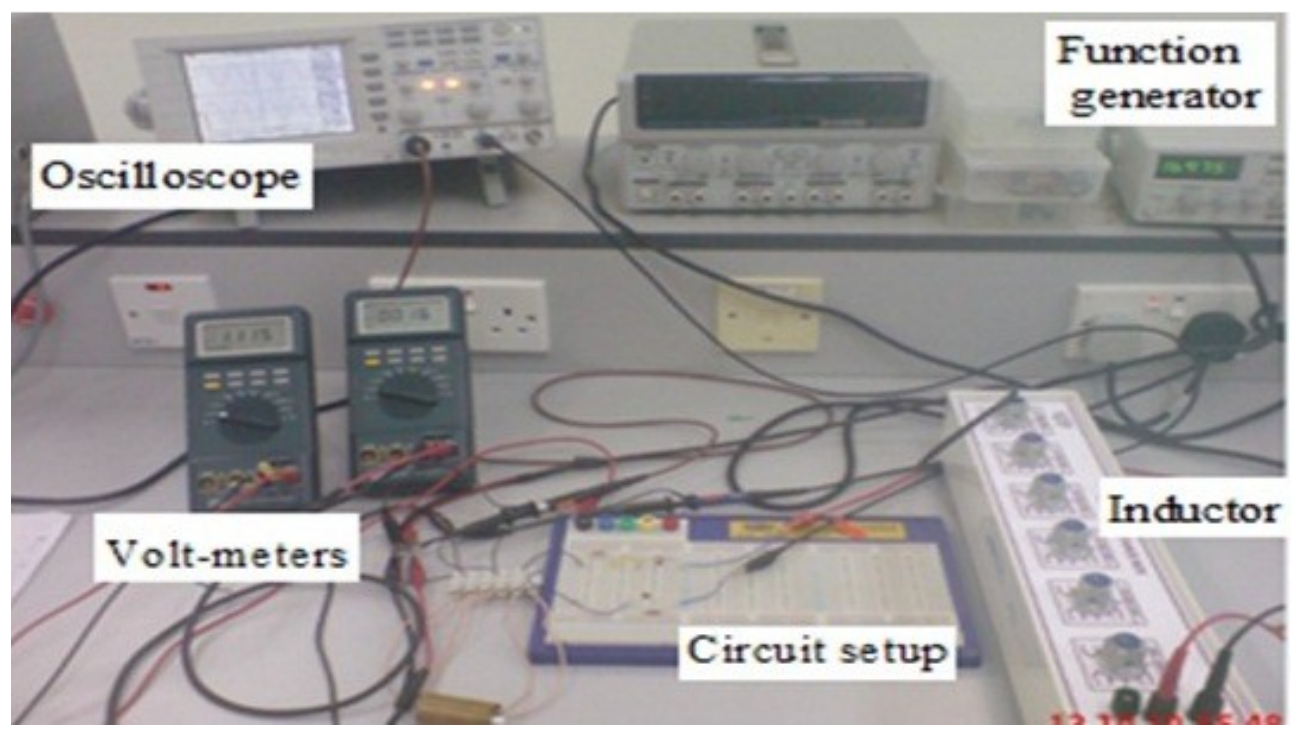

Fig. 7: Pictorial view of measuring system

Table 1:Specification of primary and secondary coils.

\begin{tabular}{|l|c|c|}
\hline \multirow{2}{*}{} & \multicolumn{2}{|c|}{ Number of turns } \\
\cline { 2 - 3 } & Coil set-1 & Coil set-2 \\
\hline Primary coil 1 & 200 & 400 \\
\hline Primary coil 2 & 200 & 400 \\
\hline Secondary coil & 1000 & 2000 \\
\hline Internal diameter (mm):10 & \\
\hline Height (mm):40 & \multicolumn{2}{|}{} \\
\hline Material of wire: copper & \multicolumn{2}{|}{} \\
\hline Thickness of wire $(\mathrm{mm}): 0.19$ &
\end{tabular}

Table 2:Model parameter for the circuit

\begin{tabular}{|l|l|c|c|}
\hline \multicolumn{1}{|c|}{ Symbol } & \multicolumn{1}{|c|}{ Description } & Value & Unit \\
\hline $\mathrm{R}_{\mathrm{x}}$ & Fixed resistor & 2 & $\Omega$ \\
\hline $\mathrm{R}_{1}$ & Series impedance in the primary coil & 50 & $\Omega$ \\
\hline $\mathrm{R}_{2}$ & Series impedance in the secondary coil & 50 & $\Omega$ \\
\hline $\mathrm{C}_{1}$ & Bypass capacitor & 220 & $\mathrm{nF}$ \\
\hline $\mathrm{C}_{2}$ & Bypass capacitor & 220 & $\mathrm{nF}$ \\
\hline
\end{tabular}




\section{RESULTS AND DISCUSSION}

Cores of different materials with the same physical dimensions were used in this work; hence the strength of the magnet will vary in accordance with the core being used. The variation in the strength is due to the varying number of flux lines passing through the core. The permeability of the material $\mu_{\mathrm{r}}$ is a measure for the strength of materials. The permeability of non-magnetic materials, such as plastic, aluminum and air is approximately the same as that of free space $\mu_{\mathrm{o}}$. Materials having permeability greater than $\mu_{0}$ are ferromagnetic (soft magnetic), such as steel. The results obtained are discussed in detail; Figures 8-11presents the result obtained by conducting the first experiment for IEUT. The coil specifications used are as stated in Table 1 (Coil-1).

Fig. 8 shows the inductive peak plot for air core when no material is inserted in the solenoid coil. A highest peak in impedance value $(8 \mathrm{kHz}, 1.402921605 \Omega)$ is achieved because there was no interruption in the electromagnetic field of the coil. Fig. 9 shows the results for plastic core. It exhibits non-magnetic and non-conductive properties similar to air, causing impedance peaks at a frequency similar to that of air $(8 \mathrm{KHz}, 0.894600647 \Omega)$. The lower permeability value of plastic compared to that of air contributes to lower inductive reactance amplitude peak. The plot in case of steel is presented in Fig. 10 where a decrease in peak value $(4 \mathrm{KHz}, 1.026549297 \Omega$ ) compared to air-core and a plastic-core plot is observed. Generally steel is a good conductor and is also considered as a magnetic material. A core of magnetic material such as steel when placed in a coil or transformer provides a better path than air or plastic for magnetic flux, thereby increasing the inductance of the coil and raising the couplingbetween windings accordingly. This in turn causes the impedance peaks at a lower value of frequency considering the circuit component value to remain the same. Accordingly the lower peak value is attributed to a lower value of permeability, assuming all other inductive reactance causing reliant factors to remain the same.Fig.11 illustrates the overall performance for the three materials used. Based on the results obtained, the materials tested appeared to exhibit distinctive and unique features of impedance peaks with varying amplitude and frequency, thus characterizing the materials under observation. Among the three materials tested so far, steel was found to be more promising that air or plastic when it comes to applications such as transducers. However, space and weight requirement priorities favor the choice of nonmagnetic materials when the sensitivity is not a pivotal issue of concern. However, in an application-specific situation, conductive material proves better than the nonconductive materials when used as a core for magneto sensor application. 


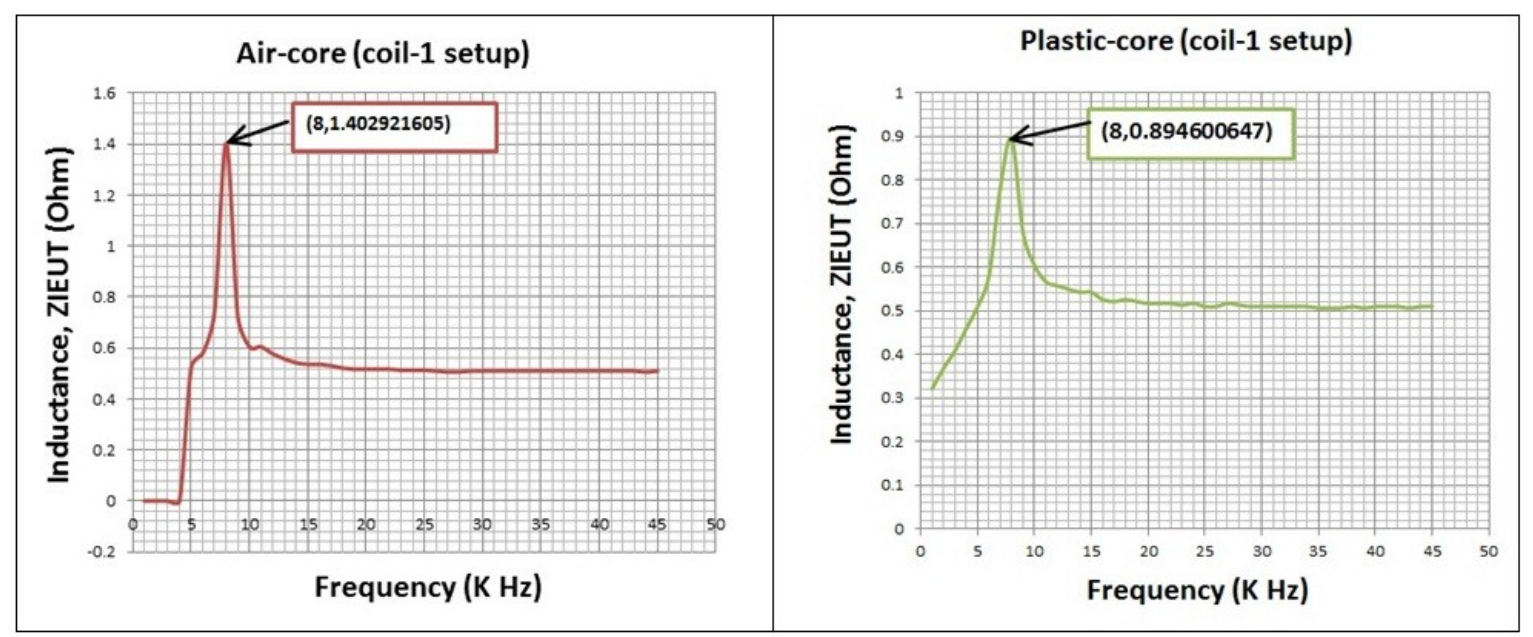

Fig.8: Inductive peak against frequency variation for air-core coil-1 setup

Fig.9:Inductive peak against frequency variation for plastic-core coil-1 setup

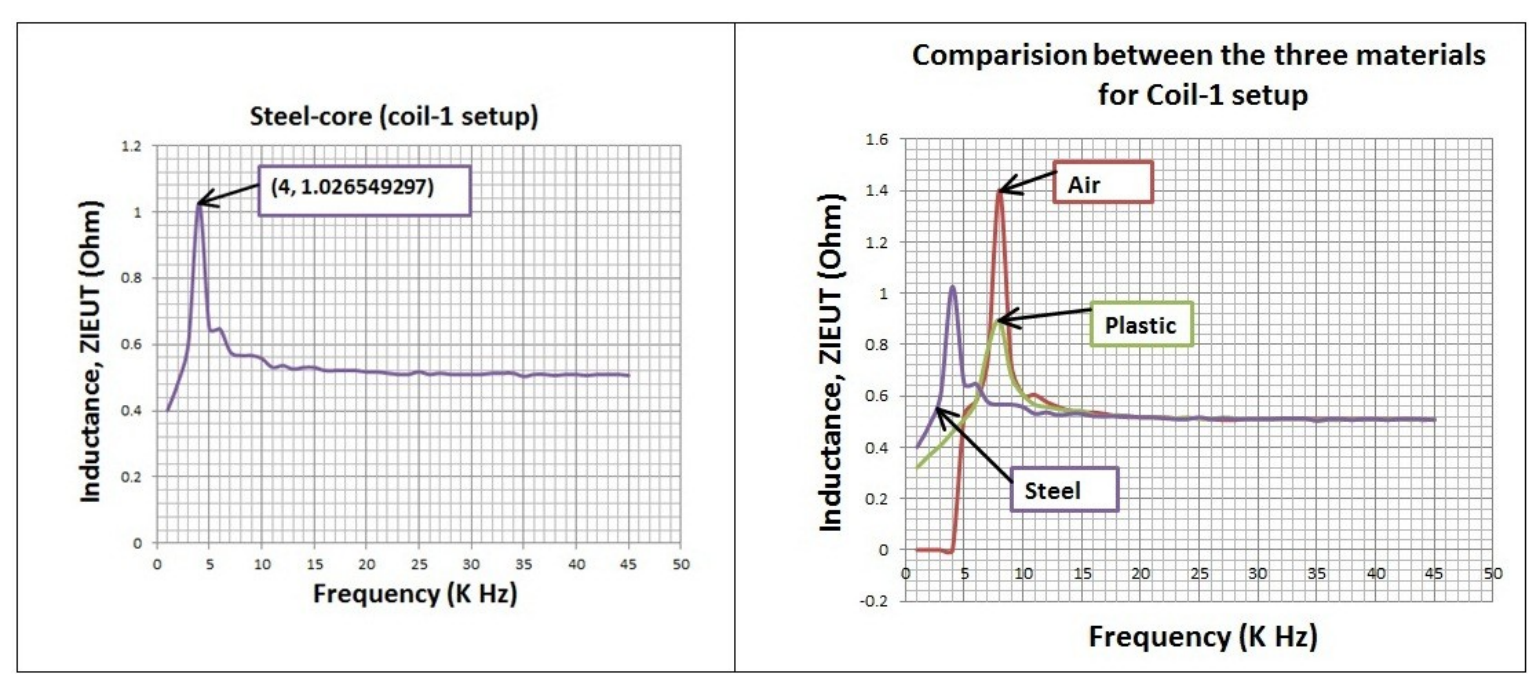

Fig.10: Inductive peak against frequency variation for steel-core coil-1 setup
Fig.11: Overall performance for the three materials with coil-1 setup 
In line with the second experiment, the second set of coil was used with an increased number of primary and secondary turns, as specified in Table 1 (Coil-2).Figures 12-15 shows the effect on the inductive peaks by the increased number of turns in a coil. The three materials under test for the new set of coils are air core, aluminium core, and steel core.

Fig.12 shows the inductive peak plot for air core when no material is inserted in the solenoid coil. Again the highest peak in impedance value for air core is achieved at 7 $\mathrm{KHZ}$, giving a high inductive peak at $1.061385 \Omega$. Fig.13 shows the inductive peak obtained for aluminium in coil-2 setup; aluminium was used instead of plastic in order to consider the inductive response for a paramagnetic material $(7 \mathrm{KHz}, 0.982917 \Omega)$. Moreover not much difference was observed in the inductive peak values, because the permeability of aluminium and plastic are relatively equal. In Fig.15 the inductive peak was obtained in the case of steel core. The inductive peak was observed at a frequency of $3 \mathrm{KHz}$ with an inductance value of $1.026549297 \Omega$. It is noteworthy that the peak value was obtained at a faster frequency response when compared with the results of coil-1 setup. Fig. 15 illustrates the overall performance of the materials with a higher number of turns in a coil. For air core, the highest peak is achieved once again owing to have no interruption in the electromagnetic field, as stated earlier. However, when an aluminium core was used, a lower peak valuewas achieved compared to steel core. This means that soft magnetic material produces a stronger magnetism compared to air and aluminium. For coil-2 setup, the number of turns was doubled, hence the magnetic field produced by each turn in the solenoid adds up, giving a stronger resultant magnetic field inside the solenoid coil. With the increased number of turns in the coil, higher voltage is induced in the coils, thus causing the inductive peak response to be faster than coil-1 setup at a lower frequency.

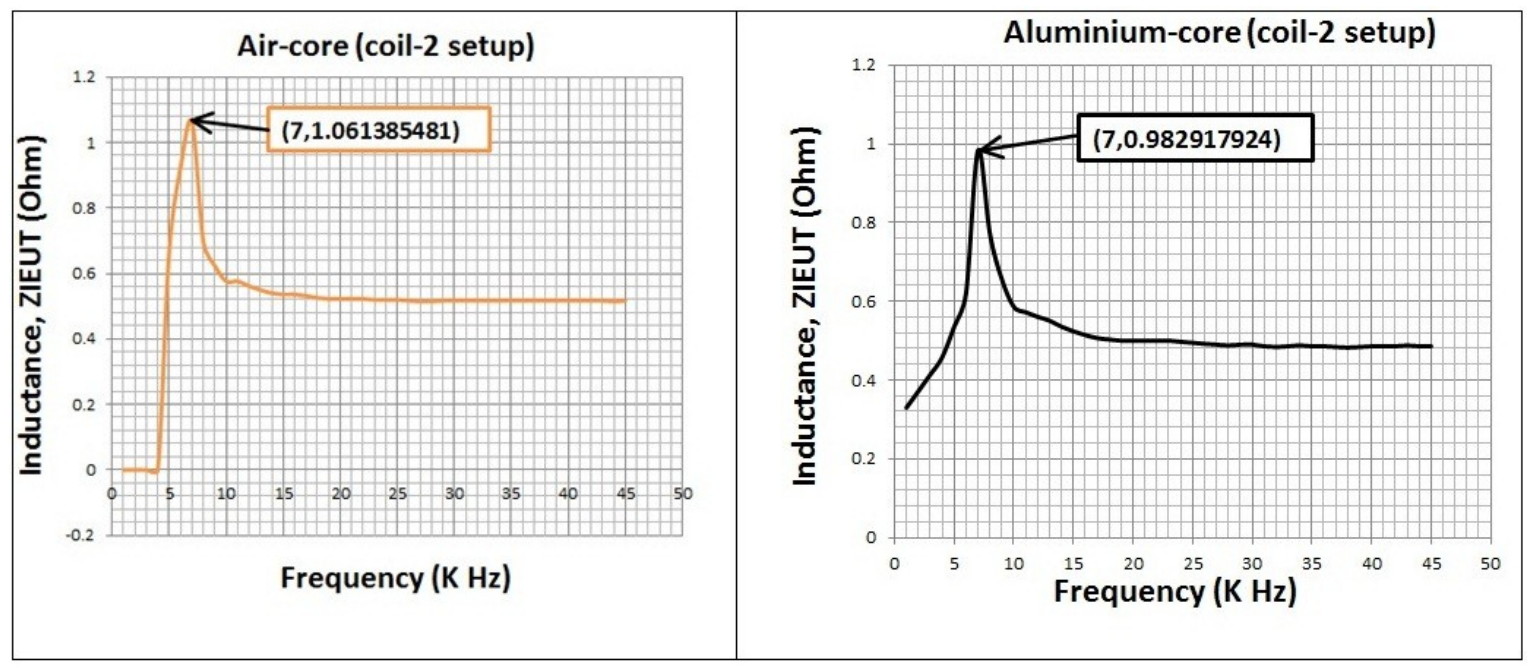

Fig.12: Inductive peak against frequency variation for air-core coil-2

Fig.13: Inductive peak against frequency variation for aluminium-core, coil-2 setup 


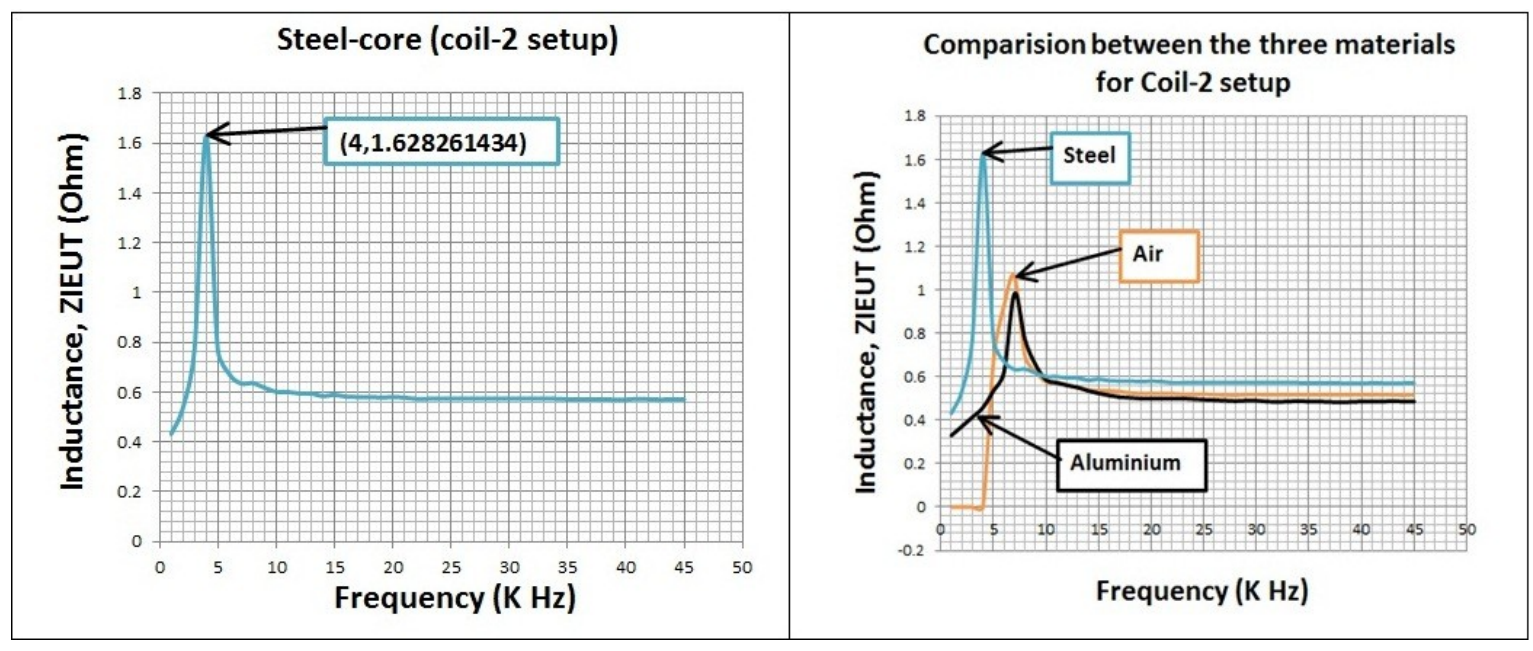

Fig.14: Inductive peak against frequency variation for steel-core coil-2 setup
Fig.15: Overall performance for the three materials with coil-2setup

With the experimental setup for resistive material under test coil-1 setup had been used with a resistive value of $30 \Omega$ and $240 \Omega$. Figures 16-19 presents the experiment results when conducted with $30 \Omega$ load.Fig.16 illustrates the resulting plots for paper core. The graph was plotted for impedance, $\mathrm{Z}_{\mathrm{MUT}}$ as a function of frequency, which ranges from 10 $\mathrm{KHz}$ to $350 \mathrm{KHz}$, by an increment of $10 \mathrm{kHz}$. Paper core was used as a reference material under test. Note that there is constant increase in the graph, till $2 \mathrm{kHz}$, it then shows a continuous decrease in the values with increasing frequency. Fig. 17 shows the impedance response for the case of aluminium. The nonmagnetic materials, aluminum and paper, showed almost similar output, since their permeability is approximately equal to of air. The graph showed shows a linear increment for a small change in frequency range. Moreover there was a constant decrement of the $\mathrm{Z}_{\text {MUT }}$ values with increasing frequency. Fig. 18 shows the impedance response obtained for the case of steel core. Steel exhibited a relatively different plot compared to paper and aluminium, as steel is a soft magnetic material. However, a frequency of $2 \mathrm{KHz}$, highest impedance peak was obtained for steel. Fig.19illustrates the results of the comparison made between the three materials under test using a $30 \Omega$ resistor. There is an overlap in the results for paper and aluminium however there is a slight variation in the plot response for steel. The results obtained using $30 \Omega$ resistor did not show a positive outcome for the purpose of characterizing materials; hence the experiment was repeated with a higher resistance load. 


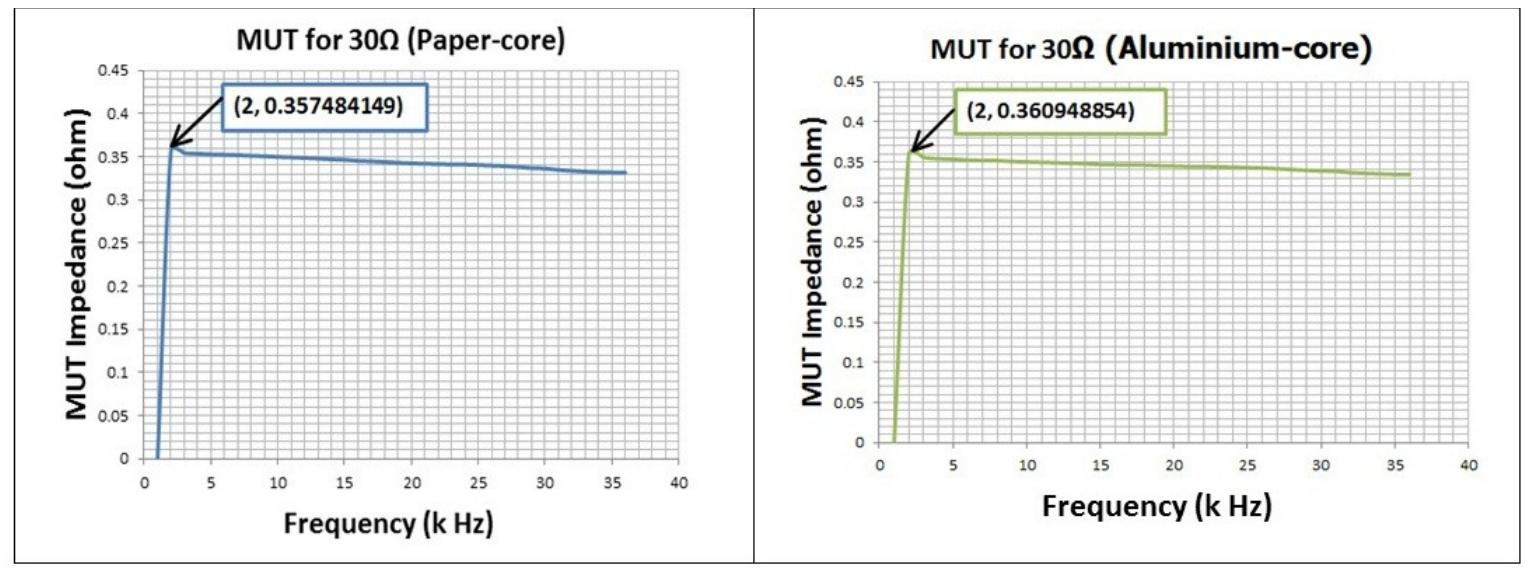

Fig.16: Resistive materials under test with a load resistance of $30 \Omega$ for papercore

Fig.17:Resistive materials under test with a load resistance of $30 \Omega$ for Aluminiumcore

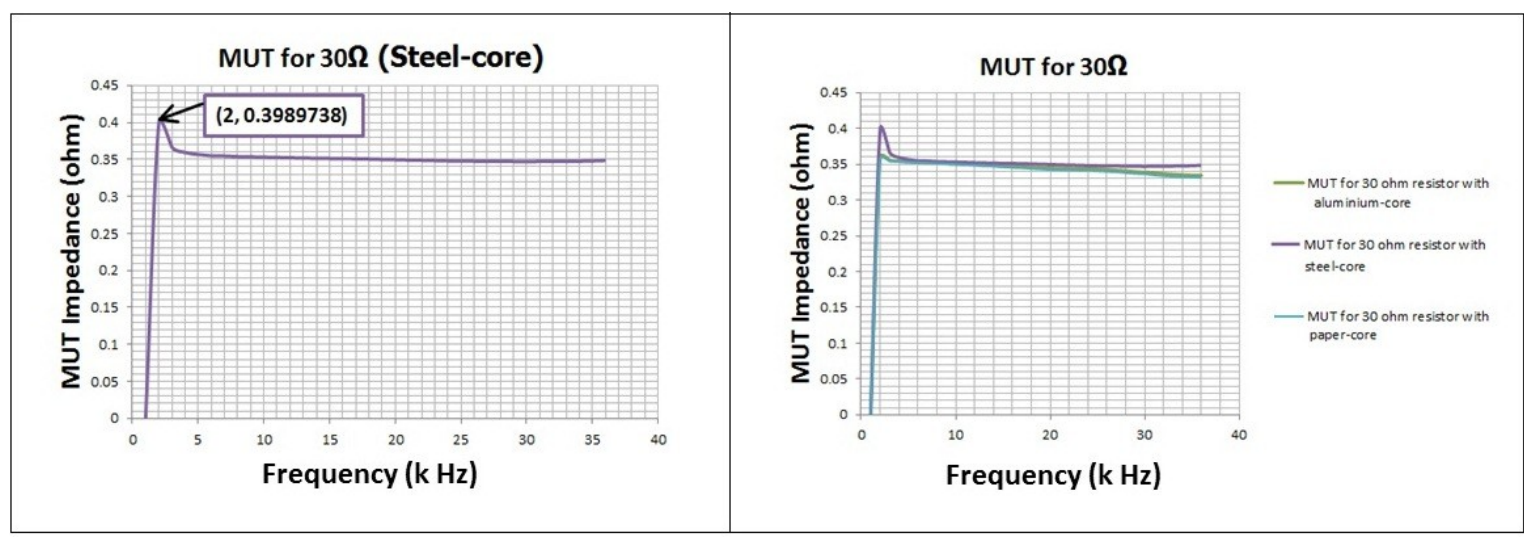

Fig.18:Resistive materials under test with a load resistance of $30 \Omega$ for steelcore
Fig.19:Overall performance for the three materials with a load resistance of $30 \Omega$

Fig. 20 shows the results for resistive material under test with a load resistance of $240 \Omega$. In the case of paper core a similar output response as in the case of $30 \Omega$ resistor was obtained. The impedance peak had resulted at a frequency of $2 \mathrm{kHz}$.Fig. 21 shows the results obtained for aluminium core condition, by using a higher resistance a very small change in the impedance peak is noted, but the frequency at which the peak occurs remains the same. Fig.22 shows the impedance response obtained for the case of steel core. Steel exhibited a higher impedance peak compared to paper and aluminium.Fig.23 illustrates the results for the comparison of the three materials under test with load resistance of $240 \Omega$. Note that not much difference was observed between the high load resistor and the low load resistor for the three resistive materials under test. The results 
almost overlap. This shows that load resistance in the main circuit is not affecting the current induction on the coil for this specific material; therefore resistors are not very applicable to be used in transducers. Moreover it can be concluded that in order to characterize materials, inductors are more well-suited than resistors.
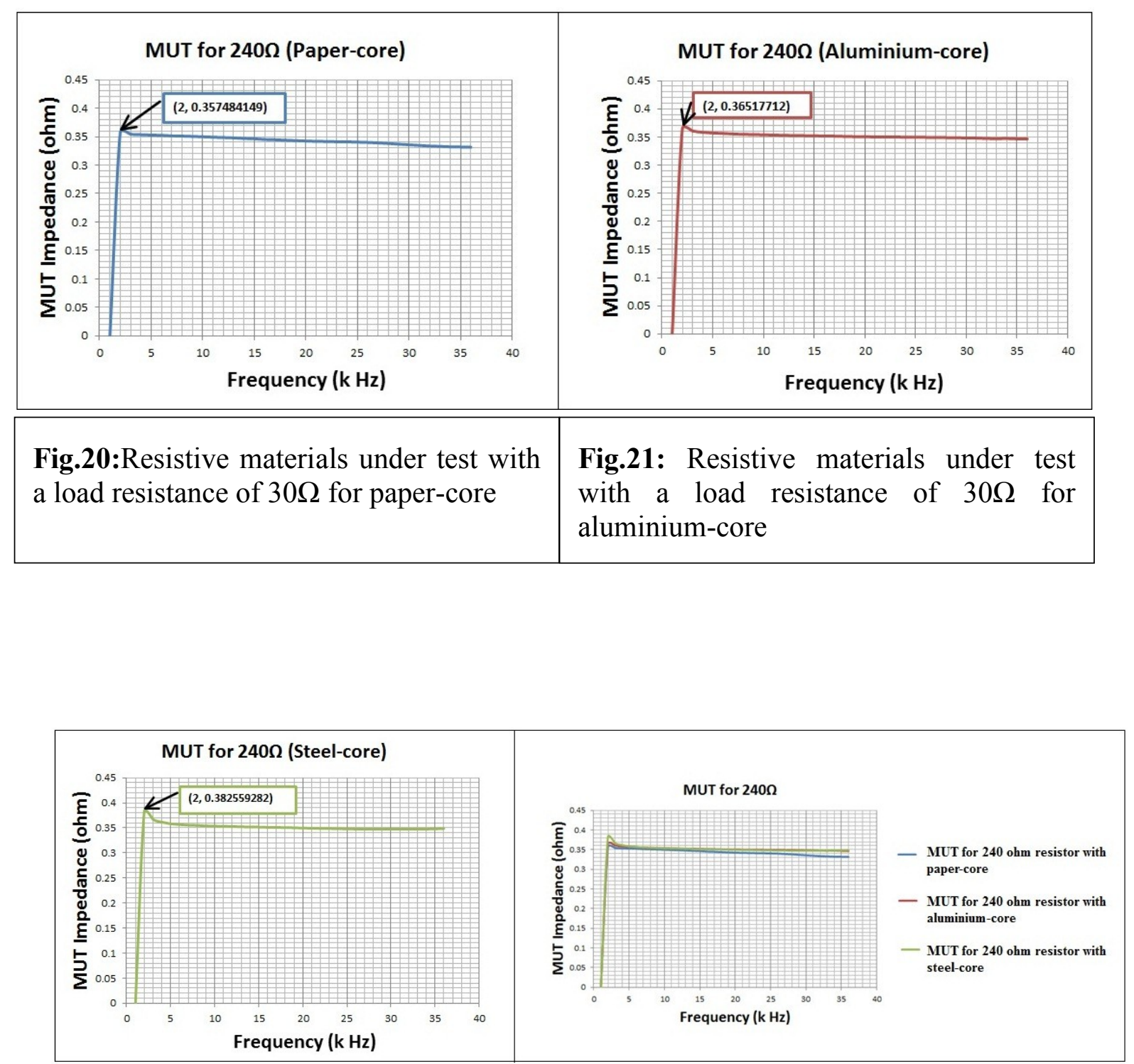

Fig.22:Resistive materials under test with a load resistance of $30 \Omega$ for steelFig.23:Overall performance for the three core materials with a load resistance of $240 \Omega$ 


\section{CONCLUSION}

In this paper, an inductive transducer sensor model is used in which magnetic and nonmagnetic materials are inserted to characterize the material by measuring the inductance of magnetic and non-magnetic inductance peaks. The characteristic of the material can be differentiated from other materials by obtaining unique distinctive impedance peaks with unique impedance values. The change in permeability of a material determines the inductance of the output coil, which is brought about by the change in position of the movable core made of the material whose inductance is measured. This paper uses a simple circuit approach by making use of inductive coupling instead of complicated IC based approaches. This novel technique for the identification and characterization of appropriate magnetic material is significantly important for applications in biomedical equipment and implants requiring low frequency. Another reason for this identification technique also comes from the particular fact that magnetic materials over the last twenty years have changed dramatically both in terms of their applications and in the magnitude of their use.

\section{REFERENCES}

[1] Lizon-Martinez, S., Tellini, B., Giannetti, R., \& Robles, G., 2009. "Measurement of Asymmetric Minor Loops in Soft Ferrites Up to Medium Frequencies". IEEE Transactions on Instrumentation and Measurement, 58(2): 423-428.

[2] Cristaldi, L., Ferrero, A., Lazzaroni, M., \&Morando A.P., 2003. "Sensorless Evaluation of Asymmetric Hysteresis Loops of Ferromagnetic Materials". IEEE Transactions on Instrumentation and Measurement, 52 (3): 846-851.

[3] Fujisaki, K., 2003. "High-Response Inductive Electromagnetic Sensor". IEEE Journal, Transactions on Magnetics, 39 (5): 2190-2193.

[4] Ferrigno, L., Liguori, C., \&Pietrosanto, A., 2002. "Measurement for the characterization of passive components in non-sinusoidal conditions". IEEE Transactions on Instrumentation and Measurement, 51 (6): 1252-1258.

[5] Weng-Yew Chang, Kye-Yak See, \& Bo Hu, 2010. "Characterization of Component Under DC Biasing Condition Using an Inductive Coupling Approach". IEEE Transactions on Instrumentation and Measurement, 59 (8): 2109-2114.

[6] Jagiella, M., Fericean, S., Droxler, R., \&Dorneich, A., 2004. "New Magneto-inductive Sensing Principle and its Implementation in Sensors for Industrial Applications". IEEE Proceeding on Sensors, 2(9): 1020-1023.

[7] XueliangHuo, Jia Wang \&Ghovanloo, M., 2008. "A Magneto-Inductive Sensor Based Wireless Tongue-Computer Interface". IEEE Transaction on Neural Systems and Rehabilitation Engineering, 16 (5): 497-504.

[8] Boukhenoufa, A.,Dolabdjin, C.P., \&Robbes, D., 2005. "High-sensitivity giant magnetoinductive magnetometer characterization implemented with a low-frequency magnetic noisereduction technique". IEEE Journal on Sensors, 5 (5): 2594-2597.

[9] Antepli, M.A., Gurbuz S. Z., \&Uysal-Biyikoglu, E., 2010. "Ferromagnetic Target Detection and Localization with a Wireless Sensor Network". Military Communications Conference, 2010-MILCOM, 1068 
1073.http://www.nve.com/Downloads/SPIE10_Magnetoresistive_Sensors_for_Nondestructiv e_Evaluation.pdfhttp://202.194.20.8/proc/MILCOM2010/papers/p1179-antepli.pdf

[10] Boukhenoufa, A., Dolabdjin, C.P., \&Robbes, D., 2005."High-sensitivity giant magnetoinductive magnetometer characterization implemented with a low-frequency magnetic noisereduction technique". IEEE Journal on Sensors, 5 (5): 2594-2597.

[11] Kangarlu, A., \&Shellock, F.G., 2000. "Aneurysm Clips: Evaluation of Magnetic Field Interactions With an 8.0 T MR System". Journal of Magnetic Resonance Imaging, 12: 107111. http://www.imrser.org/PDF/Shellock.8T.Aneurysm.JMRI.pdf

[12] Keat Ghee Ong, Ee Lim Tan, Pereles, B., \& Horton, B., 2009. :Wireless, Magnetic-Based Sensors for Biomedical Applications". IEEE Annual International Conference IEEE on Medicine and Biology Society, 5436-5439.

[13] Sheroz Khan, Jamaludin Omar, SK Khairul Hassan, AlizaAniBintiMdRalib, Ma Li Ya, AtikaArshad, AHM ZahirulAlam and MD Rafiqul Islam, 2011. "Design and Implementation of Potentiometer-Based Nonlinear Transducer Emulator". IIUM Engineering Journal 12(1): 77-87.

\section{NOMENCLATURE}

$\begin{array}{llc}A & \text { Area of coil } & \mathrm{m}^{2} \\ l & \text { Length of the coil } & \mathrm{m} \\ l-x & \text { Core displacement left from the left end } & \mathrm{m} \\ L & \text { Total inductance } & \mathrm{H} \\ L_{0} & \text { Inductance of free space } & \mathrm{H} \\ N & \text { Total number of turns } & - \\ x & \text { Core displacement fron the right end } & \mathrm{m}\end{array}$

\section{Greek letters}

$\begin{array}{llc}\mu_{\mathrm{o}} & \text { Free space permeability } & \mathrm{Hm}^{-1} \\ \mu_{\mathrm{r}} & \text { Permeability of a material } & \mathrm{Hm}^{-1}\end{array}$ 